\title{
Cardiac autonomic function in patients with myasthenia gravis: analysis of the heart-rate variability in the time-domain
}

\author{
Sherifa Ahmed Hamed ${ }^{1}$, Khaled Osama Mohamad ${ }^{1}$, Mohamad Adam² \\ ${ }^{1}$ Department of Neurology and Psychiatry, Assiut University Hospital, 71516 Assiut, Egypt. \\ ${ }^{2}$ Department of Cardiology, Assiut University Hospital, 71516 Assiut, Egypt.
}

\section{A B S T R A C T}

\begin{abstract}
Aim: Myasthenia gravis (MG) is a neuromuscular transmission disorder caused by acetylcholine receptor autoantibodies. Cardiac autonomic dysfunctions were rarely reported in patients with MG. Functional cardiac abnormalities were variable and reported in patients at severe stages of the disease and with thymoma. We investigated cardiac functions in patients with MG using Ambulatory 24-h electrocardiographic Holter-Monitoring. Methods: This study included 20 patients with MG with a mean age of $28.45 \pm 8.89$ years and duration of illness of $3.52 \pm 1.15$ years. The standard Holter reports include data for heart-rate, ventricular ectopies (VEs), supraventricular ectopies (SVEs), heart-rate variability (HRV), ST, QT, atrial fibrillation and T-wave alternans. Results: VEs, SVEs and ST-T changes were reported in $55 \%, 40 \%$ and $20 \%$ of patients respectively. Compared with healthy subjects $(n=20)$, HRV components including SDNN, SDANN, SDNN Index, RMS-SD and pNN50 ( $P=0.001$ for all) were reduced in patients indicating sympathetic and parasympathetic autonomic dysfunctions. HRV abnormalities were reported in 30-60\% of patients. No significant correlations were identified between SDNN, RMS-SD, pNN50, and duration of illness. Conclusion: Depressed HRV may be an early manifestation of autonomic neuropathy in patients with MG even in milder stages of the disease. This information is useful in rating disease progression and the efficacy of therapeutic interventions.
\end{abstract}

Key words: Cardiac autonomic function, heart-rate variability, myasthenia gravis

\section{INTRODUCTION}

Myasthenia gravis (MG) is a humoral immune attack to the skeletal muscle nicotinic acetylcholine receptors (nAChRs) at the neuromuscular junction by autoantibodies. ${ }^{[1]}$ The cardinal symptoms of MG is fatigue or fluctuating weakness in voluntary muscles with repeated or sustained exertion in the course of the day, but improved by rest. ${ }^{[1]} \mathrm{MG}$ has a prevalence of $25-125 / 10^{6}$, with female to male ratio of 3-2. ${ }^{[2]}$ About 2/3 of the patients has mild weakness, which initially involve the ocular muscles, but may progress to generalized weakness in approximately $85 \%$ of patients. Respiratory muscle weakness occurs in severe stages, which may require mechanical

\begin{tabular}{|l|l|}
\hline \multicolumn{2}{|c|}{ Access this article online } \\
\hline Quick Response Code: & \\
\hline & Website: \\
\hline & www.nnjournal.net \\
\cline { 2 - 3 } & DOI: \\
\hline
\end{tabular}

ventilation (myasthenic crisis). ${ }^{[3]}$ The diagnosis of MG was based on the association between the following parameters: clinical manifestations ${ }^{[4]}$ presence of serum anti-AChR antibody, definite clinical improvement on injection of anticholinesterase, and a decremental pattern of repetitive nerve stimulation..$^{[3]}$ The thymus gland is abnormal in up to $90 \%$ of adults with MG, of which $70 \%$ have enlarged thymic hyperplasia, whereas $10-20 \%$ have benign thymic tumors or thymoma. ${ }^{[5]}$ The current treatment for MG includes acetylcholine esterase inhibitors (AChE-Is) (as pyridostigmine), ${ }^{[6]}$ immunopharmacologic drugs, ${ }^{[7-12]}$ and thymectomy. ${ }^{[13]}$

A few studies were carried out to investigate cardiac involvement in patients with MG. Some reported nonspecific electrocardiographic (ECG) changes, Doppler imaging and conventional echocardiography, ${ }^{[14]}$ while others reported variable results, which varied from asymptomatic specific ECG changes (as abnormalities of the STI, ${ }^{[15]}$ dispersion of QT and T wave alternans $\mathrm{s}^{[16]}$, giant diffuse $\mathrm{T}$ waves, to clinically manifest abnormalities (as conduction disturbances, atrioventricular dissociation, wide QRS, ${ }^{[17]}$ syncopal 
attacks, orthostatic hypotension, impaired heart-rate variability (HRV) $)^{[18,19]}$ ). These significant cardiac manifestations were reported in patients with severe stages of MG and in the presence of thymoma. It has been suggested that some MG patients may develop autonomic dysfunction and other nervous system manifestations. ${ }^{[18-26]}$ It has also been suggested that the heart and skeletal muscle molecules are targets for the autoimmune process of $\mathrm{MG}^{\left[{ }^{[27-30]}\right.}$

This study aimed to assess cardiac functions in patients with MG using Ambulatory 24-h ECG Holter Monitoring. HRV measures are sensitive indices of cardiac autonomic function (sympathetic and parasympathetic).

\section{METHODS}

\section{Subjects}

This study included 20 (males $=6$, females $=14$ ) patients with MG without known cardiac diseases. Their age range is was 16-50 years and duration of illness ranged from 1 to 4 years. Clinical grading of the patients was done based on the medical-scientific advisory board of the MG Foundation of America classification. ${ }^{[4]}$ Patients grading was based on their histories and diagnosis shown in their medical records. Patients reported histories of weakness of ocular muscles (ptosis) (class I), with mild and predominant weakness of limb muscles (class IIa) or oropharyngeal muscles (class IIb), or with moderate and predominant weakness of limbs (class IIIa). Thymectomy was done to the seven patients with thymoma. Table 1 shows the demographic and clinical characteristics of the studied group. Patients were recruited from the Out-patient Clinic of the Department of Neurology, Assiut University Hospital, Assiut, Egypt during their follow-up visits in which they were free of clinical manifestations (i.e., after resolution of active stage of the disease for at least 3 months) and were on maintenance

\begin{tabular}{|c|c|c|c|}
\hline $\begin{array}{l}\text { Demographic and } \\
\text { clinical characteristics }\end{array}$ & $\begin{array}{l}\text { Patients } \\
(n=20)\end{array}$ & $\begin{array}{l}\text { Control subjects } \\
\quad(n=20)\end{array}$ & $\mathbf{P}$ \\
\hline Male/female & $6 / 14$ & $10 / 10$ & - \\
\hline \multirow[t]{2}{*}{ Age; years } & $16-50$ & $20-50$ & - \\
\hline & $28.45 \pm 8.89$ & $30.22 \pm 5.76$ & 0.380 \\
\hline Duration of illness; & $1-4$ & - & - \\
\hline years & $3.52 \pm 1.15$ & - & - \\
\hline \multicolumn{4}{|l|}{ Clinical grade } \\
\hline I & 0 & - & - \\
\hline $\mathrm{Ila} / \mathrm{llb}$ & $2 / 10$ & - & - \\
\hline IIla/IIIb & $8 / 0$ & - & - \\
\hline $\mathrm{IVa} / \mathrm{IVb}$ & 0 & - & - \\
\hline V & 0 & & \\
\hline \multicolumn{4}{|l|}{ Thymic pathology (\%) } \\
\hline Normal & $5(25)$ & - & - \\
\hline Hyperplasia & $8(40)$ & - & - \\
\hline Thymoma & $7(35)$ & & \\
\hline
\end{tabular}

treatment with low doses of AChE-Is and/or steroids. The patients were admitted to the hospital for $24-48 \mathrm{~h}$ for the purpose of the research. Twenty healthy subjects matched for age, sex and socioeconomic status were included in this study for statistical comparisons. Control subjects were recruited from the general population. This study was accepted by the regional Ethical Committee. All patients and control subjects were briefed about the detailed information of this study and hence consented to attend this study.

Excluded were subjects (patients and controls): (1) with respiratory involvement or in crisis (i.e., severe stage of the disease); (2) with major systemic illness such as organic heart disease, diabetes, hypertension or any other disease that might affect the autonomic nervous system; (3) on medications known to affect heart-rate/rhythm such as beta-blockers, vasopressors, digitalis, theophylline, thyroid hormones, tricyclic antidepressant, antiarrythmic drugs, atropine and its derivatives, etc.; (4) with history of febrile illness in the past 1-week; and (5) with lack of sound sleep the night prior to monitoring.

\section{Measurements}

All participants underwent conventional echocardiography and Ambulatory ECG Holter-Monitoring. Holter monitoring is the continuous 24-h monitoring of ECG activity of a patient's heart while engaged in daily activities. Ambulatory ECG was carried out using the 5-electrode Holter which is a 3-channel portable battery operated digital ECG recorder (Cardiolight FMC.A, Medset, Medizintecknik, Hamburg, Germany). The standard Holter report includes data for heart-rate, ventricular ectopies (VEs), supraventricular ectopies (SVEs), HRV, ST, QT, QTc (QT corrected for heart-rate), atrial fibrillation (A-Fib), T-wave alternans and sleep apnea.

Heart-rate variability is defined as the oscillation in the interval between consecutive heart beats as well as the oscillations between consecutive instantaneous heart-rate. ${ }^{[31]}$ The general concept of HRV is that the more the HRV, the healthier the heart, because it more readily responds to its various stimuli. Small changes in R-R (NN) variability indicate cardiac risk. However, small or large changes in variability cannot be noticed at ECG strips. Two correlated methods of calculating $\mathrm{R}-\mathrm{R}$ changes have been accepted by the cardiology community, which are the time-domain and frequency. In this study, we analyzed the HRV in the time-domain. ${ }^{[32]}$ The most acceptable measurements in time-domain are: (1) SDNN (ms): standard deviation of all qualified beats (NN intervals); (2) SDANN (ms): standard deviation of the averages of $\mathrm{NN}$ intervals in all 5-min segments of the entire recording; (3) RMS-SD (ms): 
the square root of the mean sum of squares differences between adjacent NN intervals; (4) SDNN index (ms): mean of the standard deviations of all $\mathrm{NN}$ intervals for all 5-min segments of the entire recording; (5) SDSD (ms): standard deviation of differences between adjacent NN intervals; (6) NN50 count: number of pairs of adjacent NN intervals differing by more than $50 \mathrm{~ms}$ in the entire recording; three variants are possible counting all such NN intervals pairs or only pairs in which the first or second interval is longer; and (7) pNN50\%: percentage of differences between adjacent normal NN intervals that are greater than $50 \mathrm{~ms}$ computed over the entire 24-h ECG recording.

The warning signs of cardiac problems include: VEs in excess of 10/h, VE Pair, V-Runs, R on T beats, SV-Runs, A-Fib, pauses in excess of $2.5 \mathrm{~s}$, QTc in excess of 460 ms, ST depressions of 1 millimeter or more (a strong indication of cardiac ischemia) and SDNN of $50 \mathrm{~ms}$ or less.

\section{Statistical analysis}

Calculations were done with the statistical package SPSS, version 12.0. Data were presented as mean \pm standard deviation. Unpaired two-sided Student's $t$ test was utilized for comparison among means of normally distributed parameters, while Mann-Whitney $U$-test was for all other cases. Correlations were assessed using Pearson's and Spearman's methods for normally and non-normally distributed data, respectively. For all tests, values of $P<0.05$ were considered statistically significant.

\section{RESULTS}

This study included 20 patients with MG. They had mean age of $28.45 \pm 8.89$ years and mean duration of illness of $3.52 \pm 1.15$ years. None of the patients reported symptoms related to arrhythmias as syncope, dizziness, palpitation, shortness of breath, chest discomfort, diaphoresis, or neurological symptoms such as transient ischemic attack. Conventional echocardiographic measures were normal for all patients. The total ambulatory 24-h ECG recording period was ranged between 18 and $24 \mathrm{~h}$ (mean: $22.00 \pm 0.35$ ). The minimum heart-rates were found during sleeping and ranged from 47 to 75 beats $/ \mathrm{min}$ (mean: $58 \pm 8$ ) and the maximum heart-rates trends were found during awake and ranged from 112 to 145 beats/min (mean: $90 \pm 12$ ). Minimum R-R ranged from 296 to 560 ms (mean: $421 \pm 47$ ) and the maximum $\mathrm{R}-\mathrm{R}$ intervals ranged from 868 to $1,618 \mathrm{~ms}$ (mean: $1,255 \pm 205)$. VEs and SVEs were found in 11 (55\%) and 7 (40\%) of patients (versus none for controls) respectively. SVEs were less than 25/24 h. Depressed ST-T was reported in $4(20 \%)$ patients. Table 2 shows the results of HRV in the time-domain. Patients had

\begin{tabular}{lccc}
\hline Table 2: The results of time-domain analysis of HRV & \\
Variable & Patients $(\boldsymbol{n}=\mathbf{2 0})(\%)$ & Controls $(\boldsymbol{n}=\mathbf{2 0})$ & $\mathbf{P}$ \\
\hline SDNN (ms) & $12(60)$ & 0 & 0.001 \\
& $45.30-111.20$ & $86.33-198.70$ & \\
SDANN (ms) & $55.70 \pm 20.23$ & $152.75 \pm 15.66$ & \\
& $9(45)$ & 0 & 0.001 \\
& $44.50-116.35$ & $94.40-180.68$ & \\
SDNN index (ms) & $58.19 \pm 13.80$ & $142.23 \pm 12.09$ & \\
& $12(60)$ & 0 & 0.001 \\
& $42.30-138.56$ & $92.43-166.62$ & \\
RMS-SD (ms) & $56.46 \pm 20.02$ & $112.64 \pm 16.22$ & \\
& $8(40)$ & 0 & 0.001 \\
& $12.30-45.70$ & $5.64-95.55$ & \\
pNN50 (\%) & $20.51 \pm 6.06$ & $46.57 \pm 4.24$ & \\
& $6(30)$ & 0 & 0.001 \\
& $0.10-14.50$ & $0.60-23.22$ & \\
& $1.40 \pm 0.32$ & $14.42 \pm 2.86$ & \\
\hline
\end{tabular}

Data are expressed as number of patients with abnormalities, range, mean \pm SD. SD: Standard deviation; HRV: Heart-rate variability

reduced indices of HRV (SDNN, SDANN, SDNN Index, RMS-SD and pNN50) ( $P=0.001$ for all). No significant correlations were reported between SDNN, RMS-SD, pNN50 and duration of illness.

\section{DISCUSSION}

In this study, dynamic ECG showed evidence of subclinical autonomic cardiac dysfunction in patients with MG with mild/moderate severities. Cardiac autonomic functions were assessed by measuring the cyclic variation of the heart beat intervals (i.e., HRV). HRV reflects the complex interplay of sympathetic and parasympathetic innervation of heart. The lack of intra-individual variability over time makes the measurement of HRV an excellent tool for studying autonomic input of the heart. ${ }^{[31]}$ In the present study, components of the time-domain analysis (SDNN, RMS-SD, pNN50) were significantly reduced in patients with MG. No significant correlations were identified between HRV indices and duration of illness. Accordingly, previous studies did not report significant correlations between autonomic nervous system dysfunction and disease duration, clinical manifestations, cardiovascular risk factors and diseases activity were reported. ${ }^{[19,23]}$

SDNN reflects overall autonomic modulation of the heart but does not provide no information regarding isolated sympathetic and parasympathetic activities, while RMSSD and pNN50 which reflect very short-term HRV are predominantly mediated by the vagus nerve. ${ }^{[32]}$ Reduced HRV is well known to be associated with susceptibility to cardiac arrhythmias. ${ }^{[33]}$ Previous studies had also shown that reduced HRV is an independent risk factor for arrhythmic sudden death after myocardial infarction. ${ }^{[34]}$ In their study, Vernino et al. ${ }^{[19]}$ reported impaired HRV and a low vagal tone and modified cardiac parasympathetic modulation, orthostatic hypotension and gastrointestinal manifestations (severe abdominal 
cramps, early satiety and nausea) in an adult with MG associated with thymoma. These manifestations improved by AChE-Is and thymectomy. Previous studies rarely reported other autonomic nervous system dysfunctions (sympathetic and parasympathetic) in patients with $\mathrm{MG}^{[19-22]}$ including gastrointestinal, intestinal pseudo-obstruction, ${ }^{[21,23]}$ acute autonomic and sensory neuropathy and severe panautonomic failure. ${ }^{[19,22]}$ These studies reported increase in the autonomic nervous system dysfunction with the severity of MG. Meanwhile improvement of both neuromuscular and autonomic symptoms was observed with AChE-Is and after thymectomy. Furthermore, neurophysiological tests and laboratory studies also confirm the presence of autonomic dysfunction in patients with MG. For instance, augmentation in epinephrine excretion, although the nor-epinephrine excretion remains unchanged or even undergoes reduction in response to forearm ischemia or orthostasis (a sign of sympathetic deficiency), was reported in patients with $\mathrm{MG}^{\left[{ }^{[24]}\right.}$ while both stimuli in normal subjects induce a rise in norepinephrine urinary excretion without significant changes in epinephrine excretion.

The exact mechanisms of the autonomic nervous system dysfunction in patients with MG could rely on the involvement of autonomic neuronal nAChRs by an immune mediated processes of MG. ${ }^{[25,26]}$ The structural identities between different muscle and autonomic nAChRs subunits with the possibility of cross-reactivity between different nAChRs antibodies may be contributed as a cause of immune-mediated autonomic neuropathy with MG. Additionally, a few studies reported myocarditis in patients with $\mathrm{MG}$ and suggested that cardiac involvement with MG could be due to the immune responses driven by muscle and cancer (e.g., thymoma) mediated expression of neuronal nAChRs antibodies. ${ }^{[27-30,35,36]}$ Recently, Suzuki et al. ${ }^{[22]}$ have reported myocarditis in MG patients with anti-Kv1.4 (voltage-gated potassium channel) antibodies which was further manifested by ECG findings with high frequencies of T-wave abnormality and QT prolongation, ventricular tachycardia, sick sinus syndrome, or complete atrial ventricular block and severe heart failure. These authors suggested that the heart and skeletal muscle molecules are targets for the autoimmune process of MG. ${ }^{[29]}$ Some authors suggested that autonomic nervous system manifestations of MG could result from the adverse effect of medications used for MG treatment, such as pyridostigmine. ${ }^{[37]}$ As a reversible AChE-I, pyridostigmine inhibits the enzymatic breakdown of ACh and consequently potentiating cholinergic neurotransmission with selective augmentation of the efferent parasympathetic signal. A number of studies have indicated that pyridostigmine decreases the resting heart-rate by about 5-7 beats/min and increases the R-R interval and long-term time-domain indices of HRV in normal subjects ${ }^{[38]}$ and patients with heart failure. ${ }^{[39]}$ However, none of these studies reported marked reduction of SDNN below $50 \mathrm{~ms}$ or presence of VEs, SVEs or depresses ST-T as observed in this study. We suggested that although pyridostigmine could reduce HRV indices but the marked reduction observed in this study is attributed to the autonomic nervous system involvement by the disease process.

The results of this study indicate that patients with MG are considered at high risk due to the presence of autonomic nervous system dysfunction and are candidates for earlier considerations of cardioprotective medications. Thus, caution has to be considered for prescribing drugs that disturb the cardiovascular autonomic nervous system and preanesthetic evaluation before surgery. ${ }^{[40,41]}$

Despite the strength of our findings, the main limitation of this study is the small sample size. Longitudinal and prospective studies are needed.

In conclusion, patients with MG may have specific ECG abnormalities indicating subclinical cardiac autonomic dysfunction even in milder stages of the disease. Depressed SDNN of HRV is an early warning sign of autonomic neuropathy. These results indicate the need for routine evaluation of autonomic functions in patients with MG both to identify patients at high risk for earlier consideration of cardio-protection and long-term follow up studies.

\section{REFERENCES}

1. Lindstrom JM, Seybold ME, Lennon VA, Whittingham S, Duane DD. Antibody to acetylcholine receptor in myasthenia gravis. Prevalence, clinical correlates, and diagnostic value. Neurology 1976;26:1054-9.

2. Phillips LH 2nd. The epidemiology of myasthenia gravis. Neurol Clin 1994;12:263-71.

3. Drachman DB. Myasthenia gravis. NEngl JMed 1994;330:1797-810.

4. Jaretzki A 3rd, Barohn RJ, Ernstoff RM, Kaminski HJ, Keesey JC, Penn AS, Sanders DB. Myasthenia gravis: recommendations for clinical research standards. Task Force of the Medical Scientific Advisory Board of the Myasthenia Gravis Foundation of America. Ann Thorac Surg 2000; 70:327-34.

5. Onodera $\mathrm{H}$. The role of the thymus in the pathogenesis of myasthenia gravis. Tohoku J Exp Med 2005;207:87-98.

6. Scheife RT, Hills JR, Munsat TL. Myasthenia gravis: signs, symptoms, diagnosis, immunology, and current therapy. Pharmacotherapy 1981;1:39-54.

7. Palace J, Newsom-Davis J, Lecky B. A randomized double-blind trial of prednisolone alone or with azathioprine in myasthenia gravis. myasthenia gravis study group. Neurology 1998;50:1778-83.

8. Chaudhry V, Cornblath DR, Griffin JW, O'Brien R, Drachman DB. Mycophenolate mofetil: a safe and promising immunosuppressant in neuromuscular diseases. Neurology 2001;56:94-6.

9. Drachman DB, Jones RJ, Brodsky RA. Treatment of refractory 
myasthenia: "rebooting" with high-dose cyclophosphamide. Ann Neurol 2003;53:29-34.

10. Ponseti JM, Azem J, Fort JM, Codina A, Montoro JB, Armengol M. Benefits of FK506 (tacrolimus) for residual, cyclosporin- and prednisone-resistant myasthenia gravis: one-year follow-up of an open-label study. Clin Neurol Neurosurg 2005;107:187-90.

11. Chiu HC, Chen WH, Yeh JH. The six year experience of plasmapheresis in patients with myasthenia gravis. Ther Apher 2000;4:291-5

12. Wegner B, Ahmed I. Intravenous immunoglobulin monotherapy in long-term treatment of myasthenia gravis. Clin Neurol Neurosurg 2002;105:3-8

13. Gronseth GS, Barohn RJ. Practice parameter: thymectomy for autoimmune myasthenia gravis (an evidence-based review): report of the quality standards subcommittee of the American academy of neurology. Neurology 2000;55:7-15.

14. Furlund Owe J, Skulstad Davidsen E, Eide GE, Gerdts E, Gilhus NE. Left ventricular long-axis function in myasthenia gravis. $J$ Neurol 2008;255:1777-84.

15. Ashok PP, Ahuja GK, Manchanda SC, Jalal S. Cardiac involvement in myasthenia gravis. Acta Neurol Scand 1983;68:113-20.

16. Asensio E, Gómez M, Narváez R, Castillo L, Oseguera J, Dorantes J, Orea A, Hernandez P, Rebollar V. Description of the resting electrocardiogram in a series of patients with myasthenia gravis. Rev Invest Clin 2003;55:270-5.

17. Calin C, Savu O, Dumitru D, Ghiorghiu I, Calin A, Capraru C, Popescu BA, Croitoru M, Vilciu C, Ginghina C. Cardiac involvement in myasthenia gravis - is there a specific pattern? Rom J Intern Med 2009;47:179-89.

18. Douchet MP, Quiring E, Bronner F, Vi-Fane R, Messier M, Chauvin M, Warter JM. Paradoxal lowering of parasympathetic indices in myasthenic patients. Arch Mal Coeur Vaiss 1999;92:711-7.

19. Vernino S, Cheshire WP, Lennon VA. Myasthenia gravis with autoimmune autonomic neuropathy. Auton Neurosci 2001;88:187-92.

20. de Jongste $\mathrm{MJ}$, Oosterhuis $\mathrm{HJ}$, Lie KI. Intractable ventricular tachycardia in a patient with giant cell myocarditis, thymoma and myasthenia gravis. Int $J$ Cardiol 1986;13:374-8.

21. Hofstad H, Ohm OJ, Mørk SJ, Aarli JA. Heart disease in myasthenia gravis. Acta Neurol Scand 1984;70:176-84

22. Suzuki S, Utsugisawa K, Yoshikawa H, Motomura M, Matsubara S, Yokoyama K, Nagane Y, Maruta T, Satoh T, Sato H, Kuwana M, Suzuki N. Autoimmune targets of heart and skeletal muscles in myasthenia gravis. Arch Neurol 2009;66:1334-8.

23. Suzuki S, Baba A, Kaida K, Utsugisawa K, Kita Y, Tsugawa J, Ogawa G, Nagane Y, Kuwana M, Suzuki N. Cardiac involvements in myasthenia gravis associated with anti-Kv1.4 antibodies. Eur $J$ Neurol 2014;21:223-30.

24. Senda Y, Sugimura K, Koike Y, Matsuoka Y, Takahashi A. Concurrence of acute autonomic and sensory neuropathy and myasthenia gravis - a case report and pathogenetic considerations. Rinsho Shinkeigaku 1989;29:332-5.

25. Pande R, Leis AA. Myasthenia gravis, thymoma, intestinal pseudo-obstruction, and neuronal nicotinic acetylcholine receptor antibody. Muscle Nerve 1999;22:1600-2.

26. Bogousslavsky J, Regli F, Doret AM, Fulpius BW, Ostinelli B, Rabinowicz T, Ruzicka J. Encephalopathy, peripheral neuropathy, dysautonomia, myasthenia gravis, malignant thymoma, and antiacetylcholine receptor antibodies in the CSF. Eur Neurol 1983;22:301-6.

27. Anderson NE, Hutchinson DO, Nicholson GJ, Aitcheson F Nixon JM. Intestinal pseudo-obstruction, myasthenia gravis, and thymoma. Neurology 1996;47:985-7.

28. Stoica E, Enulescu O. Deficiency of sympathetic nervous system function in myasthenia. J Auton Nerv Syst 1992;38:69-76.

29. Evoli A, Minicuci GM, Vitaliani R, Battaglia A, Della Marca G Lauriola L, Fattorossi A. Paraneoplastic diseases associated with thymoma. J Neurol 2007;254:756-62.

30. Thorlacius S, Aarli JA, Riise T, Matre R, Johnsen HJ. Associated disorders in myasthenia gravis: autoimmune diseases and their relation to thymectomy. Acta Neurol Scand 1989;80:290-5.

31. Stein PK, Bosner MS, Kleiger RE, Conger BM. Heart rate variability: a measure of cardiac autonomic tone. Am Heart $J$ 1994;127:1376-81.

32. Clinical competence in ambulatory electrocardiography. A statement for physicians from the ACP/ACC/AHA Task Force on Clinical Privileges in Cardiology. J Am Coll Cardiol 1993;22:331-5.

33. Vikman S, Mäkikallio TH, Yli-Mäyry S, Pikkujämsä S, Koivisto AM, Reinikainen P, Airaksinen KE, Huikuri HV. Altered complexity and correlation properties of R-R interval dynamics before the spontaneous onset of paroxysmal atrial fibrillation. Circulation 1999;100:2079-84.

34. Valkama JO, Huikuri HV, Koistinen MJ, Yli-Mäyry S, Airaksinen KE, Myerburg RJ. Relation between heart rate variability and spontaneous and induced ventricular arrhythmias in patients with coronary artery disease. J Am Coll Cardiol 1995;25:437-43.

35. Elgoyhen AB, Johnson DS, Boulter J, Vetter DE, Heinemann S Alpha 9: an acetylcholine receptor with novel pharmacological properties expressed in rat cochlear hair cells. Cell 1994;79:705-15.

36. Lips KS, Pfeil U, Kummer W. Coexpression of alpha 9 and alpha 10 nicotinic acetylcholine receptors in rat dorsal root ganglion neurons. Neuroscience 2002;115:1-5.

37. Lewis SW, Ron MA, Newsom-Davis J. Absence of central functional cholinergic deficits in myasthenia gravis. $J$ Neurol Neurosurg Psychiatry 1989;52:258-61.

38. Nóbrega AC, dos Reis AF, Moraes RS, Bastos BG, Ferlin EL, Ribeiro JP. Enhancement of heart rate variability by cholinergic stimulation with pyridostigmine in healthy subjects. Clin Auton Res 2001;11:11-7.

39. Behling A, Moraes RS, Rohde LE, Ferlin EL, Nóbrega AC, Ribeiro JP. Cholinergic stimulation with pyridostigmine reduces ventricular arrhythmia and enhances heart rate variability in heart failure. Am Heart J 2003;146:494-500.

40. Baraka A. Anaesthesia and myasthenia gravis. Can $J$ Anaesth 1992;39:476-86.

41. Hamed SA. Comorbid nervous system manifestations and disorders with myasthenia gravis: Evide nces and possible mechanisms. $J$ Neurol Neurosci 2012;3:2.

Cite this article as: Hamed SA, Mohamad KO, Adam M. Cardiac autonomic function in patients with myasthenia gravis: analysis of the heart-rate variability in the time-domain. Neuroimmunol Neuroinflammation 2015;2(1):21-5 Source of Support: Nil. Conflict of Interest: No.

Received: 19-08-2014; Accepted: 30-09-2014 